\title{
Resiliência em idosos atendidos na Rede de Atenção Básica de Saúde em município do nordeste brasileiro*
}

\author{
RESILIENCE AMONG THE ELDERLY CARED FOR BY THE PRIMARY HEALTHCARE \\ NETWORK IN A CITY OF NORTHEAST BRAZIL
}

\begin{abstract}
RESILIENCIA EN ANCIANOS ATENDIDOS EN LA RED DE ATENCIÓN BÁSICA DE SALUD EN MUNICIPIO DEL NORDESTE BRASILEÑO
\end{abstract}

Camomila Lira Ferreira', Lúcia Maria Oliveira Santos², Eulália Maria Chaves Maia ${ }^{3}$

\section{RESUMO}

Objetiva-se avaliar a capacidade de resiliência, a auto-estima e o apoio social em idosos através de um estudo exploratório com uma amostra por conveniência constituída por idosos usuários da rede pública de saúde do município de Natal-RN, Brasil, os quais responderam ao questionário e às Escalas de Resiliência, Auto-estima e Apoio Social. Participaram 65 idosos com idade média de 71 anos, sendo $81 \%$ do sexo feminino, com baixas condições sócioeconômicas. Identificou-se que os idosos apresentam características resilientes, autoestima positiva e apoio social percebido como satisfatório, mesmo diante das perdas e declínios vivenciados na velhice. Além disso, verificaram-se correlações moderadas e positivas entre resiliência e auto-estima, já que na medida em que uma aumenta, a outra também se eleva. Assim, o ato de viver bem esse período crítico parece ser favorecido pela resiliência enquanto recurso propiciador do desenvolvimento e pelos fatores de proteção, importantes indicadores de saúde.

\section{DESCRITORES}

Idoso

Envelhecimento

Resiliência psicológica

Saúde do idoso

\begin{abstract}
The objective of this study is to evaluate the capacity of resilience, self-esteem and social support among the elderly by means of an exploratory study. The convenience sample consisted of elderly users of the public healthcare network in NatalRN, Brazil, who answered the questionnaire and the Resilience, Self-Esteem and Social Support Scales. Participants were 65 seniors, with a mean age of 71 years, $81 \%$ female, with poor socio-economic conditions. It was found that the elderly participants have resilient characteristics, positive self-esteem and social support perceived as satisfactory, despite their losses and decline due to aging. Furthermore, moderate and positive correlations were observed between resilience and self-esteem, because as one increased, the other also increased. Therefore, it appears that resilience, considered a resource that assists development, favors staying well through this critical period, as well as the protective factors, which are important health indicators.
\end{abstract}

DESCRIPTORS
Aged
Aging
Resilience, psychological
Health of the elderly

\begin{abstract}
RESUMEN
Se objetiva evaluar la capacidad de resiliencia, autoestima y apoyo social en ancianos mediante estudio exploratorio con muestra por conveniencia constituida por ancianos, pacientes de la red pública de salud del municipio de Natal/RN-Brasil, los cuales respondieron al cuestionario y a las Escalas de Resiliencia, Autoestima y Apoyo Social. Participaron 65 ancianos, edad promedio 71 años, $81 \%$ de sexo femenino, con baja condición socioeconómica. Se identificó que los ancianos presentan características resilientes, autoestima positiva y apoyo social percibido como satisfactorio, aún ante las pérdidas y declinaciones experimentadas en la vejez. Además de eso, se verificaron correlaciones moderadas $y$ positivas entre resiliencia y autoestima, ya que en la medida en que una aumenta, la otra también se eleva. De este modo, vivir bien dicho período crítico parece ser favorecido por la resiliencia como recurso favorecedor del desarrollo y por los factores de protección, importantes indicadores de salud.
\end{abstract}

\section{DESCRIPTORES \\ Anciano \\ Envejecimiento \\ Resiliencia psicológica \\ Salud del anciano}

\footnotetext{
* Extraído da pesquisa "Resiliência e velhice: a influência de fatores de risco e de proteção no processo de envelhecimento", Universidade Federal do Rio Grande do Norte, 2008. ${ }^{1}$ Psicóloga. Mestranda do Programa de Pós-Graduação em Ciências da Saúde da Universidade Federal do Rio Grande do Norte. Especialização em Psicologia da Saúde, Desenvolvimento e Hospitalização. Pesquisadora Voluntária do Grupo de Estudos Psicologia e Saúde do Departamento de Psicologia da Universidade Federal do Rio Grande do Norte. Rio Grande do Norte, RN, Brasil. camomilapsi@yahoo.com.br ${ }^{2}$ Graduanda do Curso de Psicologia da Universidade Federal do Rio Grande do Norte. Bolsista de Iniciação Científica do GEPS. Natal, RN, Brasil aldenor_lucia@yahoo.com.br ${ }^{3}$ Psicóloga. Professora Doutora do Departamento de Psicologia e do Programa de Pós-Graduação da Universidade Federal do Rio Grande do Norte. Coordenadora do Grupo de Estudos Psicologia e Saúde. Natal, RN, Brasil.geps_ufrn@hotmail.com
}

$\begin{aligned} \text { Rev Esc Enferm USP } & \text { Recebido: } 18 / 02 / 2010 \\ \text { 2012; 46(2):328-34 } & \text { Aprovado: 27/09/2011 }\end{aligned}$




\section{INTRODUÇÃO}

A partir da compreensão de resiliência enquanto capacidade humana de se adaptar e transformar situações de risco e de vulnerabilidade em potencialidades, este artigo procura evidenciar a importância de entender como alguns idosos, apesar de todas as desvantagens da veIhice, conseguem aceitar e viver com saúde essa fase do desenvolvimento, minimizando os efeitos de suas dores e perdas acumuladas ao longo da vida.

Nesta perspectiva, a pesquisa buscou avaliar a capacidade de resiliência, a auto-estima e o apoio social em idosos, verificando como estes fatores de proteção estão envolvidos no processo de envelhecimento dos participantes deste estudo.

\section{REVISÃO DE LITERATURA}

A resiliência é um conceito que cada vez mais vem sendo discutido na literatura científica internacional e nacional. Ela é compreendida como a capacidade humana de enfrentar as adversidades, proporcionando ao indivíduo ser transformado por esses fatores potencialmente estressores, adaptando-se ou superando tais experiências traumáticas e/ou estressantes. A resiliência é discutida não apenas como um atributo inato ou adquirido, mas sim um processo interativo e multifatorial, envolvendo aspectos individuais, o contexto ambiental, a quantidade e qualidade dos eventos vitais, e a presença dos fatores de proteção ${ }^{(1)}$.

Os fatores de proteção se constituem em características potenciais na promoção de resiliência, uma vez que podem minimizar os eventuais efeitos negativos ou disfuncionais na presença do risco, além da possibilidade de modificar, melhorar ou alterar a resposta pessoal diante de um perigo qualquer. Assim, os fatores de proteção não necessariamente eliminam os riscos, mas encorajam o indivíduo a superá-los, atuando como mediadores e protetores da adversidade ${ }^{(1-2)}$.

Ao considerar a resiliência como uma capacidade para se desenvolver normalmente sob condições difíceis ou de risco, e que todas as pessoas, em menor ou maior intensidade, terão que enfrentar alguma dessas condições enquanto estiverem vivos, a resiliência e a interação entre os fatores de risco e de proteção podem se integrar ao longo de toda a vida humana, seja na infância, na adolescência, na fase adulta ou na velhice ${ }^{(2)}$.

O estudo desse constructo entre os idosos passou a ter mais relevância na medida em que houve uma maior preocupação em virtude do envelhecimento populacio- nal. Isso se deve ao aumento da expectativa de vida que, segundo a Organização Mundial de Saúde (OMS), será de 73 anos no ano de 2025. O mesmo ocorrerá no Brasil, cuja expectativa de vida passará de 67 anos para 74 anos em 2025 , ocasionando a duplicação no número de idosos no país que atualmente corresponde a mais de 18 milhões de indivíduos com mais de 60 anos de idade ${ }^{(3)}$.

Quanto ao envelhecimento, inúmeras definições procuram caracterizar esse processo do desenvolvimento humano. A maioria dos conceitos refere-se à multideterminação desse processo, assinalado por modificações em seus aspectos físicos, sociais, biológicos e psicológicos, e dimensões políticas, culturais e econômicas. Em meio a isso, tem-se a velhice como uma etapa do ciclo vital frequentemente vista como um período de estagnação e finalização da vida, de declínio e de perdas, de desespero e temor à morte, sem possibilidades de crescimento, participação e envolvimento nos mais variados contextos ${ }^{(4)}$.

O envelhecimento humano se configura, portanto, como um processo do ciclo evolutivo que pode oferecer riscos ao bem-estar psicológico e à boa qualidade de vida, uma vez que é acompanhado por perdas significativas para o indivíduo que está envelhecendo. Todas as mudanças por quais passam os idosos podem resultar na vivência de sentimentos negativos, tais como abandono, inutilidade, falta de autonomia, falta de controle sobre o meio e sobre si mesmo ${ }^{(4)}$. Porém, na medida em que diversos fatores biológicos, sociais, psicológicos, culturais e econômicos influenciam o processo de envelhecimento humano, é importante observar que diferentes aspectos agem na vida do idoso com o objetivo de protegê-lo das situações de risco e/ou condições adversas vivenciadas, de mobilizá-lo para adaptação e/ou superação dessas vivências, de fazê-lo aprender novos conteúdos e compensar possíveis perdas ${ }^{(1)}$.

Percebe-se que, mesmo na presença de ameaças e dos riscos inerentes à velhice, alguns idosos podem se desenvolver normalmente e evitar a ocorrência de patologias diversas, sem complicações ou sequelas que dificultam sua independência e sua autonomia. Dessa forma, o idoso, após superar uma situação de risco, passa a ter uma participação ativa em seu tratamento e um envolvimento na prevenção de condições crônicas e seus sintomas. Isso se faz possível ao reverter as perdas associadas à idade. Para isso, essa população faz uso de recursos como a capacidade de resiliência, o atributo pessoal da auto-estima, o apoio e a assistência da família, de equipes de saúde e instituições da comunidade ${ }^{(5)}$. Assim, por meio desses recursos, os idosos têm possibilidade de enfrentar positivamente as adversidades que marcam essa fase do desenvolvimento, obtendo êxito no decorrer do processo de envelhecimento. 


\section{MÉTODO}

Nesse estudo exploratório que objetivou avaliar a resiliência na velhice em uma amostra de conveniência constituída por 65 idosos usuários dos serviços oferecidos pela Rede Básica de Atenção à Saúde do Distrito Sanitário Leste do município de Natal/RN, Brasil.

Para isso, utilizou-se um questionário estruturado, contendo questões sócio-demográficas, bem como uma Escala de Resiliência(6), adaptada para o português ${ }^{(1)}$. Esta escala é um dos poucos instrumentos usados para medir níveis de adaptação psicossocial positiva em face de eventos de vida importantes ${ }^{(1)}$, já que abarca as principais características da resiliência, sendo de fácil aplicação e entendimento para qualquer faixa etária e nível de escolaridade. Possui 25 itens descritos de forma positiva com resposta tipo likert variando de 1 (nada característico) a 7 (totalmente característico). Os escores da escala oscilam de 25 a 175 pontos, com valores altos indicando elevada resiliência.

Para investigar os fatores de proteção houve o auxílio da Escala Apoio Social adaptada para o português ${ }^{(7)}$. Esta escala permite a investigação do apoio social e suas associações com desenlaces relacionados à saúde em um grupo populacional no Brasil, através da avaliação de cinco dimensões de apoio social: material (provisão de recursos práticos e ajuda material); emocional (apoio que envolva confiança, disponibilidade em ouvir, compartilhar preocupações/medos e compreender seus problemas), de informação (com o recebimento de sugestões, bons conselhos, informação e conselhos desejados), afetiva (demonstração de afeto e amor, dar um abraço e amar) e de interação social positiva (diversão juntos, relaxar, fazer coisas agradáveis e distrair a cabeça) ${ }^{(7)}$. Nessa escala, os altos escores associam-se a um apoio social satisfatório.

Outro fator de proteção foi estudado com a Escala Auto-Estima $^{(8)}$, adaptada para o português ${ }^{(9)}$. A referida escala tem sua aplicabilidade indicada, contendo 10 itens designados a avaliar globalmente a atitude positiva ou negativa de si mesmo. Nela, os altos escores associam-se a uma alta auto-estima.

Foram incluídos no estudo aqueles voluntários com 60 anos ou mais de idade que apresentaram suas funções mentais preservadas, estando orientados no tempo e no espaço, além de possuírem capacidade de verbalização oral e que consentissem, livre e esclarecidamente, em responder aos protocolos da pesquisa. Aqueles que apresentaram seu estado mental comprometido ou que não concordaram com os procedimentos propostos não participaram ou foram excluídos da pesquisa. Tal avaliação da capacidade de orientação temporal e espacial ocorreu através da aplicação do Mini-Exame do Estado Mental ${ }^{(10)}$. A pesquisa foi apreciada e aprovada pelo Comitê de Ética em Pesquisas da Universidade Federal do Rio Grande do Norte (CEP-UFRN), sob Protocolo no 022/06 - CEP-UFRN, sendo tomados todos os preceitos éticos previstos na Resolução no 196/96, do Conselho Nacional de Saúde, que permitiram aos idosos responderem aos protocolos, com a utilização da técnica da entrevista estruturada em situação individual, em um só encontro.

Considerando a importância da complementaridade das informações obtidas, as escalas foram corrigidas conforme suas orientações específicas e seus escores, juntamente com os dados do questionário, foram organizados e analisados utilizando o Software Statistical Package for the Social Sciences (SPSS) 12.0. Para a análise dos dados, foi utilizada a estatística descritiva e o teste de correlação de Pearson que mensura o grau da correlação (e a direção dessa correlação - se positiva ou negativa) entre duas variáveis de escala métrica (intervalar ou de rácio). E para determinar a confiabilidade dos resultados obtidos em cada escala utilizada, optou-se pela verificação do Coeficiente Alfa de Cronbach, de acordo com a proposta de George e Mallery ${ }^{(11)}$ que possui a seguinte regra heurística para validação do coeficiente alfa: a > 0.9 igual a excelente, a $>0.8$ igual a bom, a $>0.7$ igual a aceitável, a $>0.6$ igual a questionável, a $>0.5$ igual a pobre, a $<0.5$ igual a inaceitável.

\section{RESULTADOS}

Observou-se que $83 \%(N=54)$ são do sexo feminino, com idade média de 71 anos (DP=7). Na amostra, 40\% $(\mathrm{N}=26)$ são viúvos e $66 \%(\mathrm{~N}=43)$ apresentam até 5 anos de estudo (Tabela 1). Quanto à renda familiar, $80 \%(\mathrm{~N}=52)$ da amostra possui entre 1 e 3 salários-mínimos, provenientes de suas aposentadorias ou pensões, as quais sustentam em média 3,8 pessoas ( $D P=2,1$ ) em suas casas (Tabela 1 ).

Tabela 1 - Descrição da amostra de idosos usuários da Rede de Atenção Básica de Saúde do Distrito Sanitário Leste - Natal, RN, Brasil - 2009

\begin{tabular}{|c|c|c|c|}
\hline Variável & & $\mathbf{n}$ & $\%$ \\
\hline Situação & Casado(a)/Com companheiro(a) & 25 & 39 \\
\hline \multirow[t]{3}{*}{ Conjugal } & Viúvo & 26 & 40 \\
\hline & Separado(a)/Divorciado(a) & 10 & 15 \\
\hline & Solteiro(a) & 4 & 6 \\
\hline \multirow[t]{3}{*}{ Idade (anos) } & 60 a 69 & 33 & 51 \\
\hline & 70 a 79 & 25 & 39 \\
\hline & 80 ou mais & 7 & 10 \\
\hline \multirow[t]{4}{*}{ Escolaridade } & Até 5 anos de estudo & 43 & 66 \\
\hline & De 5 a 8 anos de estudo & 6 & 9 \\
\hline & De 8 a 11 anos de estudo & 3 & 5 \\
\hline & Nunca estudou & 13 & 20 \\
\hline \multirow{4}{*}{$\begin{array}{l}\text { Renda } \\
\text { Familiar }\end{array}$} & Até 3 salários-mínimos & 53 & 81 \\
\hline & De 4 a 6 salários-mínimos & 8 & 12 \\
\hline & De 7 a 9 salários-mínimos & 2 & 3 \\
\hline & De 10 a 12 salários-mínimos & 2 & 3 \\
\hline Convivência & 01 Geração & 11 & 17 \\
\hline \multirow[t]{4}{*}{ Geracional } & 02 Gerações & 27 & 42 \\
\hline & 03 Gerações & 24 & 37 \\
\hline & 04 Gerações & 2 & 3 \\
\hline & 05 Gerações & 1 & 1 \\
\hline Total & & 65 & 100 \\
\hline
\end{tabular}

Resiliência em idosos atendidos na Rede de Atenção Básica de Saúde em município do nordeste brasileiro Ferreira CL, Santos LMO, Maia EMC 
Dentre as atividades realizadas pelos idosos desse estudo, $83 \%(\mathrm{~N}=54)$ realizam funções domésticas diariamente, 57\% ( $N=37)$ têm atividades de lazer, 91\% ( $N=59)$ usufruem de atividades de entretenimento como assistir televisão e ouvir rádio, $83 \%(\mathrm{~N}=54)$ frequentam atividades religiosas, $83 \%(\mathrm{~N}=54)$ não participam de atividades educativas e $51 \%(N=33)$ não realizam esportes. No que se refere à avaliação que os idosos fazem de seus relacionamentos, eles referiram, em sua maioria, bons relacionamentos interpessoais (Tabela 2).

Tabela 2 - Auto-avaliação de relacionamentos interpessoais de idosos usuários da Rede de Atenção Básica de Saúde do Distrito Sanitário Leste - Natal, RN, Brasil - 2009

\begin{tabular}{llcc}
\hline Variável & & N & \% \\
\hline $\begin{array}{l}\text { Relacionamento } \\
\text { com } \\
\text { companheiro(a) }\end{array}$ & $\begin{array}{l}\text { Não possui } \\
\text { companheiro }\end{array}$ & 40 & 61 \\
& Péssimo/Ruim & 1 & 1 \\
& Regular & 5 & 8 \\
& Bom/Ótimo & 19 & 30 \\
\hline Relacionamento & Não possui filho(s) & 8 & 12 \\
com filho(s) & Péssimo/Ruim & 2 & 3 \\
& Regular & 15 & 23 \\
& Bom/Ótimo & 40 & 62 \\
\hline Relacionamento & Não possui neto(s) & 12 & 19 \\
com neto(s) & Péssimo/Ruim & 1 & 1 \\
& Regular & 13 & 20 \\
& Bom/Ótimo & 39 & 60 \\
\hline Relacionamento & Não possui & 8 & 12 \\
com amigo(s) & amigo(s) & & \\
& Regular & 5 & 8 \\
& Bom/Ótimo & 52 & 80 \\
\hline Total & & 65 & 100 \\
\hline
\end{tabular}

Com a correção da escala de resiliência, identificou-se o escore médio foi indicador de um alto escore de resiliência (Figura 1). Quanto aos resultados obtidos com a escala de auto-estima, o escore médio obtido também foi um indicador de um alto escore (Figura 2).

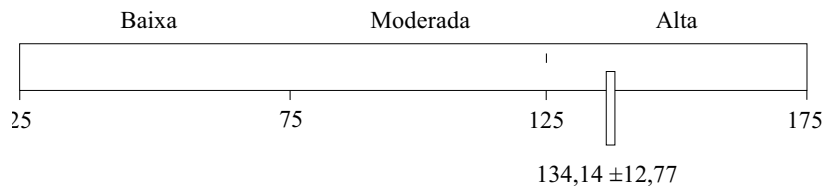

Figura 1 - Resiliência em idosos usuários da Rede de Atenção Básica de Saúde do Distrito Sanitário Leste - Natal, RN, Brasil - 2009

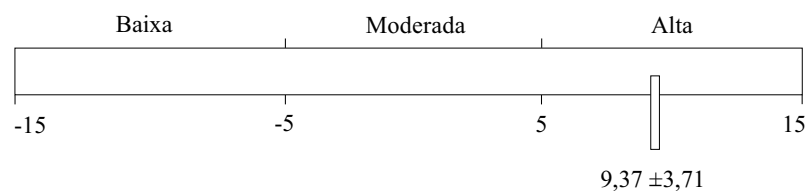

Figura 2 - Auto estima em idosos usuários da Rede de Atenção Básica de Saúde do Distrito Sanitário Leste - Natal, RN, Brasil -2009
No que diz respeito à escala de apoio social aplicada neste estudo, os resultados são apresentados por dimensões, cada uma sendo avaliação por escores finais que vão de 0 a 100. Desta forma, na dimensão material do apoio social percebido, obteve-se o escore médio de 90,85 \pm 14,65; na dimensão afetiva, o escore médio de $93,95 \pm$ 13,18 ; na emocional, a média de $80,62 \pm 20,00$; na dimensão de informação, foi de $79,85 \pm 21,97$; e na interação social positiva, $82,62 \pm 20,00$.

Ao correlacionar a resiliência e os fatores de proteção estudados, identificou-se, por meio do coeficiente de Pearson, uma correlação moderada e positiva entre os escores de auto-estima e de resiliência $(r=0,410)$. Entre as variáveis resiliência e apoio social, não houve correlação significativa. No entanto, entre as dimensões de apoio social, o que se percebeu foram correlações moderadas e positivas entre a dimensão de apoio social de informação e a dimensão de apoio social emocional $(r=0,640)$, entre as dimensões de apoio social afetivo e de interação social positiva $(r=0,595)$, e entre as dimensões de apoio social de interação social positiva e emocional $(r=0,546)$.

Através da verificação do Coeficiente Alfa de Cronbach, observou-se que a Escala de Resiliência apresentou a = 0,7360, já na Escala de Apoio Social foi a $=0,7930$ e na Escala de Auto-Estima, $a=0,7416$. Tais coeficientes indicam que a confiabilidade dos resultados obtidos é considerada como aceitável.

\section{DISCUSSÃO}

A partir deste estudo, identificou-se a predominância de mulheres na amostra, confirmando a tendência apresentada em outros estudos brasileiros ${ }^{(12-13)}$. Essa predominância refletiu a maior longevidade das mulheres em relação aos homens - um fenômeno que tem sido atribuído, a uma menor exposição a determinados fatores de risco, uma menor prevalência de tabagismo e uso de álcool, a diferenças quanto à atitude em relação a doenças e incapacidades, e a uma maior cobertura da assistência gineco-obstétrica ${ }^{(13)}$.

A idade média encontrada está um pouco acima da faixa etária mais comumente observada nos estudos brasileiros ${ }^{(12)}$, embora seja uma média que caracteriza os idosos denominados mais jovens, ou seja, aqueles com idade abaixo de 80 anos. Esses idosos denominados mais jovens pelos referidos autores apresentam uma elevada plasticidade o que não acontece com os idosos denominados muito idosos, ou seja, aqueles que apresentam 80-85 anos acima, na qual a capacidade adaptativa se reduz gradualmente ${ }^{(13)}$.

No que se refere à baixa escolaridade encontrada, esta é justificada pela restrição da Educação para a pequena elite brasileira. A escolaridade é considerada um fator de proteção associado ao bem-estar psicológico, já que quanto mais anos de estudo do indivíduo, maiores as competências desse indivíduo para o alcance de satisfa- 
ção com a vida e de equilíbrio dos afetos ${ }^{(14)}$. Os poucos anos de estudo dos idosos também influenciam no baixo nível sócio-econômico evidenciado nesse estudo, cujos proventos dos idosos advêm, em sua maioria, de aposentadorias, pensões ou benefícios governamentais, o que está de acordo com outros estudos ${ }^{(13,15)}$.

Quase a totalidade da amostra reside em domicílios multigeracionais, característico das áreas periféricas na América Latina, de todas as regiões do país ${ }^{(13)}$ e do distrito sanitário estudado que apresenta baixa renda familiar. Esses autores acreditaram que residir com duas ou mais gerações pode representar um arranjo de sobrevivência necessário para filhos e netos desses idosos. Além disso, quanto maior o número de pessoas morando no domicílio do idoso, melhor a afetividade e o auxílio que esse idoso recebe ${ }^{(17)}$.

Percebe-se avaliações positivas que os idosos fazem dos relacionamentos que possuem com seus filhos, netos e amigos. Evidencia-se, dessa forma, que os idosos não se isolam, mas sim constituem uma rede positiva e diversificada de relacionamentos fortemente associada com desfechos positivos para os idosos, como algumas medidas de saúde e no enfrentamento das mudanças decorrentes do processo de envelhecimento ${ }^{(17)}$.

Os idosos parecem ocupar bem seu tempo diário com o engajamento em diversas atividades, com destaque às atividades de entretenimento para auxiliar no preenchimento do tempo ocioso, às atividades domésticas mais comuns entre as mulheres e às atividades religiosas tidas como refúgio para as perdas e problemas de saúde. A presença de idosos mais jovens na amostra provavelmente propicia esse envolvimento em atividades variadas e sociais, bem como o envolvimento das mulheres em atividades domésticas parece ser influenciado pelo que a cultura define como mais adequado e aceitável|(15). 0 pequeno envolvimento em atividades de lazer pode estar relacionado à menor escolaridade e ao baixo nível socioeconômico da amostra, o que dificulta o acesso a interações destinadas ao lazer disponibilizado pela sociedade atual ${ }^{(17)}$. $O$ envolvimento com a religiosidade na velhice corresponde, por vezes, a uma estratégia de enfrentamento eficaz e a Igreja surge, nesse sentido, como um forte elemento na rede de apoio social do idoso, quer seja através da promoção da fé quer seja pela possibilidade de estabelecer novos vínculos positivos com os demais frequentadores desta Instituição.

Ao avaliar a resiliência nessa população, identificouse que esses idosos estão enfrentando a velhice de forma resiliente, estando de acordo com a concepção de que os idosos têm grande capacidade de resiliência e, frente a perdas, tendem a organizar seu ambiente de maneira a maximizar afetos positivos e a minimizar os negativos. Considerando as possibilidades de crescimento na velhice, a resiliência pode fazer com que os idosos sejam positivos em circunstâncias difíceis, desafios e exigências inerentes ao processo de envelhecimento ${ }^{(18)}$.
A auto-estima parece estar favorecendo esse potencial denominado resiliência nos idosos, já que se apresentou como favorável nos resultados encontrados nesse estudo e pode ter funcionado como um recurso psicológico útil para o idoso. Nesse sentido, uma auto-estima satisfatória corresponde a um fator protetor que auxilia no enfrentamento dos desafios adaptativos na velhice, bem como está fortemente relacionada à saúde, ao bem-estar e à felicidade, ajudando a amenizar os efeitos negativos do estresse e a aumentar a longevidade ${ }^{(19)}$.

Outra variável estudada e que facilita esse processo de enfrentamento e a capacidade adaptativa é o apoio social, avaliado, em termos médios, como adequado pelos idosos, em todas as suas dimensões estudadas. Alguns autores ${ }^{(16-17)}$ acreditam que idosos com uma rede social de apoio mais desenvolvida e com mais recursos psicológicos e sociais usam estratégias de adaptação mais ricas e saudáveis ao desenvolvimento, além de conseguirem neutralizar os efeitos das desvantagens da velhice e manter satisfatória sua qualidade de vida, suas auto-concepções e seu ajustamento pessoal. Fatores estudados também se relacionam ao apoio social disponível ao idoso e percebidos nessa amostra, tais como: os relacionamentos positivos com amigos e familiares, a participação em grupos, as residências multigeracionais, o estado civil de casado ou viúvo, e ter filhos ${ }^{(17)}$.

No que diz respeito à resiliência e à auto-estima constatou-se que elas tiveram uma correlação positiva, uma vez que, na medida em que uma aumenta, a outra também sofre elevação. Assim, percebeu-se que os idosos da amostra, embora se encontrem em um momento do desenvolvimento marcado por adversidades, parecem estar se adaptando bem a todas as mudanças inerentes ao envelhecimento. Tal fato pode indicar que um desenvolvimento satisfatório não está relacionado apenas à idade, mas sim a aspectos multifatoriais, entre eles o enfrentamento e a aceitação de sua condição de vida(20-21).

Dessa forma, a auto-estima é um fator de proteção importante para o desenvolvimento do idoso, já que está relacionada com a saúde e o bem-estar psicológico, e com ela estão implicados outros elementos, tais como apoio social, auto-eficácia e autonomia, que influenciarão na forma como os idosos viverão sua velhice ${ }^{(19,22)}$. Assim, embora seja uma fase de significativas mudanças, ela também é de desenvolvimento, com perspectivas positivas na trajetória do idoso ${ }^{(18,21-22)}$.

\section{CONCLUSÃO}

Assim, a resiliência e os fatores de proteção encontrados nessa pesquisa propiciam uma vivência saudável dessa fase do desenvolvimento marcada por perdas e ganhos, na medida em que se observa, através dos resultados encontrados, que os idosos pesquisados conseguiram superar as adversidades que surgiram em suas vidas até $o$
Resiliência em idosos atendidos na Rede de Atenção Básica de Saúde em município do nordeste brasileiro Ferreira CL, Santos LMO, Maia EMC 
momento, pois a resiliência é uma maneira de sobreviver relacionada à integridade, adaptabilidade e tenacidade, principalmente quando favorecida por elementos protetivos como a auto-estima e o apoio social também presentes nessa amostra. Portanto, a partir dos dados apresentados percebe-se, entre outros aspectos, que a resiliência independe de algumas variáveis, o que leva a pensar que a resiliência não depende de traços e disposições pessoais apriorísticos, nem se manifesta apenas a partir da superação de fatores de risco predeterminados. A resiliência corresponde, nesse sentido, a um processo normativo da adaptação, presente na espécie humana e aplicável ao desenvolvimento em ambientes favoráveis ou adversos. Pertence, portanto, a todos os seres vivos essa capacidade para o desenvolvimento saudável e positivo. Nessa perspectiva, torna-se fundamental a elaboração de ações que favoreçam a promoção da resiliência, com estratégias que promovam a auto-estima, já que este construto está associado à resiliência nesse estudo, representando um importante indicador de saúde mental e nas análises sociais de desenvolvimento. Com isso, pode-se favorecer o bem-estar social e individual tão almejado para os idosos nesse período de desenvolvimento e de adaptação.

Dessa forma, verifica-se a necessidade de compreender a velhice com um período do ciclo vital que merece

\section{REFERÊNCIAS}

1. Pesce RP, Assis SG, Avanci JQ, Santos NC, Malaquias JV, CarvaIhaes R. Adaptação transcultural, confiabilidade e validade da escala de resiliência. Cad Saúde Pública. 2005;21(2):436-48.

2. Leipold B, Greve W. Resilience: a conceptual bridge between coping and development. Eur Psychol. 2009;14(1):40-50.

3. Instituto Brasileiro de Geografia e Estatística (IBGE). Síntese de Indicadores Sociais. Rio de Janeiro; 2006. (Informação Demográfica e Socioeconômica, 19).

4. Lamond AJ, Deep CA, Allison M, Langer R, Reichstadt J, Moore DJ, et al. Measurement and predictors of resilience among community-dwelling alder women. J Psychiatry Res. 2009;43(1):148-54.

5. Neri AL. Palavras-chave em gerontologia. Campinas: Alínea; 2005.

6. Wagnild GM, Young HM. Development and psychometric evaluation of resilience scale. J Nurs Meas. 1993;1(2):165-78.

7. Chor D, Griep RH, Lopes CS, Faerstein E. Medidas de rede e apoio social no estudo pró-saúde: pré-testes e estudo piloto. Cad Saúde Pública. 2001;17(4):887-96.

8. Rosenberg M. Society and the adolescent self-image. Princeton: Princeton University Press; 1989. atenção dos profissionais, das políticas e dos serviços de saúde. Com isso, pode-se promover o que se denomina de envelhecimento bem-sucedido, na medida em que são desenvolvidos, para o idoso, programas que visem à manutenção de suas capacidades funcionais proporcionando, assim, uma melhoria na auto-estima e, consequentemente, na sua qualidade de vida. Tais programas devem apresentar caráter interdisciplinar e se fundamentar na implantação de alternativas de participação, ocupação e convívio do idoso com as demais gerações, dando-lhes condições para lidar com as demandas do dia-a-dia e favorecendo mecanismos que propiciem o desenvolvimento saudável.

Como limitação deste estudo, destaca-se o fato de ser transversal, representando um recorte da amostra estudada, e também acabou não sendo possível estabelecer outras relações significativas, tais como entre a resiliência e as variáveis sócio-demográficas estudadas, e entre estas últimas e a auto-estima e o apoio social. No entanto, espera-se que este estudo possa ter reunido informações que contribuam para ampliar os atuais níveis de conhecimento na área sobre resiliência no enveIhecimento humano, e que os resultados possam servir como referencial para futuras pesquisas, auxiliando os profissionais da saúde e a população no que se refere aos cuidados com idosos.

9. Avanci S, Assis S, Santos N, Oliveira R. Adaptação transcultural da Escala de Autoestima de Rosenberg para adolescentes. Psicol Reflex Crit. 2007;20(3):397-405.

10. Folstein MF, Folstein SE, McHugh PR. Mini-mental state. A practical method for grading the cognitive state of patients for the clinician. J Psychiatry Res. 1975;12(1):189-98.

11. George D, Mallery P. SPSS for windows step by step: simple guide and reference. Boston: Ally \& Bacon; 2003.

12. Moraes JFD, VBA. Factors associated with the successful aging of the socially-active elderly in the metropolitan region of Porto Alegre. Rev Bras Psiquiatr. 2005;27(4):302-8.

13. Coelho Filho JM, Ramos LR. Epidemiologia do envelhecimento no nordeste do Brasil: resultados de inquérito domiciliar. Rev Saúde Pública. 1999;33(5):445-53.

14. Queroz NC, Neri AL. Bem-estar psicológico e inteligência emocional entre homens e mulheres na meia-idade e na veIhice. Psicol Reflex Crit. 2005;18(2):292-9.

15. Ng TP, Broekman BFP, Niti M, Gwee X, Kua EH. Determinants of successful aging using a multidimensional definition among Chinese elderly in Singapore. Am J Geriatr Psychiatry. 2009;17(5):407-16. 
16. Pinto JLG, Garcia ACO, Bocchi SCM, Carvalhaes MABL. Características do apoio social oferecido a idosos de área rural assistida pelo PSF. Ciênc Saúde Coletiva. 2006;11(3):753-64.

17. Rabelo DF, Neri AL. Recursos psicológicos e ajustamento pessoal frente à incapacidade funcional na velhice. Psicol Estud. (Maringá). 2005;10(3):403-12.

18. Fortes TFR, Portuguez MW, Argimon IIL. A resiliência em idosos e sua relação com variáveis sociodemográficas e funções cognitivas. Estud Psicol (Campinas). 2009;26(4):455-63.

19. Collins AL, Smyer MA. The resilience of self-esteem in late adulthood. J Aging Health. 2005;17(4):471-89.
20. Noronha MGRCS, Cardoso PS, Moraes TNP, Centa ML. Resiliência: nova perspectiva na promoção da saúde da família? Ciênc Saúde Coletiva. 2009;14(2):497-506.

21. Berardinelli LMM, Santos MLSC. Oficina pedagógica de enfermagem: uma experiência da convergência cuidado-educação. Rev Gaúcha Enferm. 2007;28(3):430-8.

22. Freitas MC, Queiroz TA, Sousa JAV. The meaning of old age and the aging experience of in the elderly. Rev Esc Enferm USP [Internet]. 2010 [cited 2010 July 15];44(2):403-8. Available from: http://www.scielo.br/pdf/reeusp/v44n2/ en_24.pdf

\section{Agradecimentos}

À Coordenação de Aperfeiçoamento de Pessoal de Nível Superior (CAPES) pela bolsa de mestrado cedida à primeira autora deste artigo. Aos alunos de iniciação científica da base de pesquisa Grupo de Estudos: Psicologia e Saúde do Departamento de Psicologia da Universidade Federal do Rio Grande do Norte. 